\title{
On applications of Caputo $k$-fractional derivatives
}

\author{
Ghulam Farid ${ }^{1 *}$, Naveed Latif², Matloob Anwar ${ }^{3}$, Ali Imran ${ }^{1}$, Muhammad Ozair ${ }^{1}$ and Madeeha Nawaz
}

"Correspondence:

faridphdsms@hotmail.com;

ghlmfarid@cuiatk.edu.pk

${ }^{1}$ Department of Mathematics,

COMSATS University Islamabad, Attock, Pakistan

Full list of author information is available at the end of the article

\begin{abstract}
This research explores Caputo $k$-fractional integral inequalities for functions whose $n$th order derivatives are absolutely continuous and possess Grüss type variable bounds. Using Chebyshev inequality (Waheed et al. in IEEE Access 7:32137-32145, 2019) for Caputo $k$-fractional derivatives, several integral inequalities are derived. Further, Laplace transform of Caputo $k$-fractional derivative is presented and Caputo $k$-fractional derivative and Riemann-Liouville $k$-fractional integral of an extended generalized Mittag-Leffler function are calculated. Moreover, using the extended generalized Mittag-Leffler function, Caputo $k$-fractional differential equations are presented and their solutions are proposed by applying the Laplace transform technique.
\end{abstract}

Keywords: Chebyshev inequality; k-Riemann-Liouville fractional integrals; Caputo fractional derivatives; Caputo $k$-fractional derivatives; Fractional differential equations

\section{Introduction}

Fractional calculus is the study of fractional order derivatives and integrals. It has gained extensive attention of the researchers in the last few decades. It has exceptional applications in diverse fields of science and engineering. In this context, Riemann-Liouville fractional integrals and fractional derivatives are the basis of fractional calculus [7]. From the occurrence of the definition of Riemann-Liouville fractional integrals and derivatives, the authors started to think and defined fractional formulas which are extensions and generalizations of Riemann-Liouville fractional integrals and derivatives. For example, Caputo gave an improved fractional derivative formula known as Caputo fractional derivative [2].

The aim of this study is to analyze a $k$-analogue definition of Caputo fractional derivatives given in (1.8) and (1.9), and an extended generalized Mittag-Leffler function (1.18) in the prospect of Grüss type inequalities and generalized fractional differential equations.

In the following we provide needful definitions of fractional integrals and fractional derivatives along with some formulae.

Definition 1 ([7]) Let $g \in L_{1}[a, b]$. Then the Riemann-Liouville fractional integrals of order $\alpha \in \mathbb{C}(\operatorname{Re}(\alpha)>0)$ are defined by

$$
R_{a+}^{\alpha} g(x)=\frac{1}{\Gamma(\alpha)} \int_{a}^{x}(x-t)^{\alpha-1} g(t) d t, \quad x>a,
$$

(c) The Author(s) 2019. This article is distributed under the terms of the Creative Commons Attribution 4.0 International License (http://creativecommons.org/licenses/by/4.0/), which permits unrestricted use, distribution, and reproduction in any medium, provided you give appropriate credit to the original author(s) and the source, provide a link to the Creative Commons license, and indicate if changes were made. 
and

$$
R_{b-}^{\alpha} g(x)=\frac{1}{\Gamma(\alpha)} \int_{x}^{b}(t-x)^{\alpha-1} g(t) d t, \quad b>x .
$$

These integrals are called the left-sided and the right-sided Riemann-Liouville fractional integrals respectively. Here $\Gamma(\cdot)$ is the gamma function and $R_{a+}^{0} g(t)=R_{b-}^{0} g(t)=g(t)$.

Riemann-Liouville fractional derivatives are defined as follows.

Definition 2 ([7]) Let $\alpha \in \mathbb{C}, \operatorname{Re}(\alpha) \geq 0, n=[\operatorname{Re}(\alpha)]+1$, and $g \in L_{1}[a, b]$. Then the leftsided and right-sided Riemann-Liouville fractional derivatives of order $\alpha$ are defined by

$$
\left(D_{a+}^{\alpha} g\right)(x)=\frac{1}{\Gamma(n-\alpha)}\left(\frac{d}{d x}\right)^{n} \int_{a}^{x} \frac{g(t)}{(x-t)^{\alpha-n+1}} d t, \quad x>a
$$

and

$$
\left(D_{b-}^{\alpha} g\right)(x)=\frac{1}{\Gamma(n-\alpha)}\left(-\frac{d}{d x}\right)^{n} \int_{x}^{b} \frac{g(t)}{(t-x)^{\alpha-n+1}} d t, \quad x<b .
$$

In particular, if $\alpha=n \in \mathbb{N}$, then $\left(D_{a+}^{0} g\right)(x)=\left(D_{b-}^{0} g\right)(x)=g(x),\left(D_{a+}^{n} g\right)(x)=g^{(n)}(x)$, and $\left(D_{b-}^{n} g\right)(x)=(-1)^{n} g^{(n)}(x)$, where $g^{(n)}(x)$ is the usual derivative of order $n$ of the function $g(x)$.

Definition 3 ([10]) Let $\alpha \in \mathbb{R}^{+}$and $n \in \mathbb{N}$ such that $n-1<\alpha<n, g \in L_{1}[a, b]$. Then the leftsided and right-sided $k$-Riemann-Liouville fractional integrals of function $g$ are defined by

$$
R_{k, a}^{\alpha} g(x)=\frac{1}{k \Gamma_{k}(\alpha)} \int_{a}^{x}(x-t)^{\frac{\alpha}{k}-1} g(t) d t, \quad x>a,
$$

and

$$
R_{k, b}^{\alpha} g(x)=\frac{1}{k \Gamma_{k}(\alpha)} \int_{x}^{b}(t-x)^{\frac{\alpha}{k}-1} g(t) d t, \quad x<b,
$$

where $\Gamma_{k}(\cdot)$ is the $k$-gamma function defined as follows (see [3]):

$$
\Gamma_{k}(\alpha)=\int_{0}^{\infty} t^{\alpha-1} e^{\frac{-t^{k}}{k}} d t
$$

also $\Gamma_{k}(\alpha+k)=\alpha \Gamma_{k}(\alpha)$. For $k=1$, (1.5) and (1.6) give the definition of Riemann-Liouville fractional integrals and (1.7) provides Euler gamma function $\Gamma(\cdot)$.

Caputo fractional derivatives are defined as follows.

Definition 4 ([2]) Let $\alpha>0$ and $\alpha \notin\{1,2,3, \ldots\}, n=[\alpha]+1, g \in A C^{n}[a, b]$, the space of functions having $n$th derivatives absolutely continuous. Then the left-sided and rightsided Caputo fractional derivatives of order $\alpha$ are defined by

$$
\left(C_{a+}^{\alpha} g\right)(x)=\frac{1}{\Gamma(n-\alpha)} \int_{a}^{x} \frac{g^{(n)}(t)}{(x-t)^{\alpha-n+1}} d t, \quad x>a,
$$


and

$$
\left(C_{b-}^{\alpha} g\right)(x)=\frac{(-1)^{n}}{\Gamma(n-\alpha)} \int_{x}^{b} \frac{g^{(n)}(t)}{(t-x)^{\alpha-n+1}} d t, \quad x<b
$$

If $\alpha=n \in\{1,2,3, \ldots\}$ and the usual derivative $g^{(n)}(x)$ of order $n$ exists, then the Caputo fractional derivative $\left(C_{a+}^{\alpha} g\right)(x)$ coincides with $g^{(n)}(x)$, whereas $\left(C_{b-}^{\alpha} g\right)(x)$ coincides with $g^{(n)}(x)$ with exactness to a constant multiplier $(-1)^{n}$. In particular, we have

$$
\left(C_{a+}^{0} g\right)(x)=\left(C_{b-}^{0} g\right)(x)=g(x)
$$

where $n=1$ and $\alpha=0$.

Definition 5 ([5]) Let $\alpha>0, k \geq 1$, and $\alpha \notin\{1,2,3, \ldots\}, n=[\alpha]+1, g \in A C^{n}[a, b]$. Then the left-sided and right-sided Caputo $k$-fractional derivatives of order $\alpha$ are defined by

$$
\left(C_{k, a+}^{\alpha} g\right)(x)=\frac{1}{k \Gamma_{k}\left(n-\frac{\alpha}{k}\right)} \int_{a}^{x} \frac{g^{(n)}(t)}{(x-t)^{\frac{\alpha}{k}-n+1}} d t, \quad x>a,
$$

and

$$
\left(C_{k, b-}^{\alpha} g\right)(x)=\frac{(-1)^{n}}{k \Gamma_{k}\left(n-\frac{\alpha}{k}\right)} \int_{x}^{b} \frac{g^{(n)}(t)}{(t-x)^{\frac{\alpha}{k}-n+1}} d t, \quad x<b .
$$

If $\alpha=n \in\{1,2,3, \ldots\}$ and the usual derivative $g^{(n)}(x)$ of order $n$ exists, then the Caputo $k$ fractional derivative $\left(C_{1, a+}^{n} g\right)(x)$ coincides with $g^{(n)}(x)$, whereas $\left(C_{1, b-}^{n} g\right)(x)$ coincides with $g^{(n)}(x)$ with exactness to a constant multiplier $(-1)^{n}$.

In particular, we have

$$
\left(C_{1, a+}^{0} g\right)(x)=\left(C_{1, b-}^{0} g\right)(x)=g(x)
$$

where $n, k=1$ and $\alpha=0$. For $k=1$, Caputo $k$-fractional derivatives give the definition of Caputo fractional derivatives.

In [4], the following definition of Caputo $k$-fractional derivatives for a convolution of two functions is studied and some interesting results have been established. This also behaves as a generalization of Caputo $k$-fractional derivatives [12].

Definition 6 The right-sided and the left-sided Caputo $k$-fractional derivatives of convolution $g * h$ of two functions $g$ and $h$ are defined by

$$
\left(C_{k, a+d}^{\alpha} g * h\right)(x)=\frac{1}{k \Gamma_{k}\left(n-\frac{\alpha}{k}\right)} \int_{a}^{x} \frac{g^{(n)}(t) h^{(n)}(t)}{(x-t)^{\frac{\alpha}{k}-n+1}} d t, \quad x>a,
$$

and

$$
\left(C_{k, b-b}^{\alpha} g * h\right)(x)=\frac{(-1)^{n}}{k \Gamma_{k}\left(n-\frac{\alpha}{k}\right)} \int_{x}^{b} \frac{g^{(n)}(t) h^{(n)}(t)}{(t-x)^{\frac{\alpha}{k}-n+1}} d t, \quad x<b .
$$


For $k=1$, the above expressions give the definition of Caputo fractional derivatives of convolution $g * h$ of two functions $g$ and $h$. The following result has been proved in [12].

Lemma 1 Let $\alpha>0, k \geq 1$, and $\alpha \notin\{1,2,3, \ldots\}, n=[\alpha]+1$. If $g(x)=(x-a)^{p}$, where $p \geq n$, then the left-sided Caputo $k$-fractional derivative is given by

$$
\left(C_{k, a+}^{\alpha}(x-a)^{p}\right)=\frac{(x-a)^{p-\frac{\alpha}{k}} k^{\frac{\alpha}{k}-1} \Gamma(p+1) \Gamma_{k}(n k-\alpha)}{\Gamma_{k}\left(n-\frac{\alpha}{k}\right) \Gamma_{k}(p k-\alpha+k)} .
$$

From the above lemma one can get some of the derivatives as follows:

(i) $\left(C_{k, 0^{+}}^{1 / 2}(x)^{p}\right)=\frac{k^{\frac{1}{2 k}-p-1} \Gamma(p+1) x^{p-\frac{1}{2 k}}}{\Gamma\left(\frac{2 k(p+1)-1}{2 k}\right)}$.

(ii) $\left(C_{k, 0^{+}}^{1 / 2}(x)\right)=\frac{k^{\frac{1}{2 k}-2} \times x^{1-\frac{1}{2 k}}}{\Gamma\left(\frac{4 k-1}{2 k}\right)}$.

(iii) $\left(C_{1,0^{+}}^{1 / 2}(x)^{p}\right)=\frac{\Gamma(p+1) x^{p-\frac{1}{2}}}{\Gamma\left(\frac{2 p+1}{2}\right)}$.

(iv) $\left(C_{1,0^{+}}^{1 / 2}(x)\right)=\frac{2 \sqrt{x}}{\sqrt{\pi}}$.

The following Chebyshev inequality has been proved in [12].

Theorem 1 Letf $:[0, \infty) \rightarrow \mathbb{R}$ and $g:[0, \infty) \rightarrow \mathbb{R}$ be the two functions. If $n$th derivatives of $f$ and $g$ have the same monotonicity, then for Caputo $k$-fractional derivatives the following inequality holds:

$$
\left(C_{k, a+}^{\alpha} g * h\right)(x) \geq \frac{k \Gamma_{k}\left(n-\frac{\alpha}{k}+k\right)}{(x-a)^{n-\frac{\alpha}{k}}}\left(C_{k, a+}^{\alpha} g\right)(x)\left(C_{k, a+}^{\alpha} h\right)(x)
$$

For some more recent results for Caputo $k$-fractional derivatives, see $[6,8,11]$.

Next we give the definition of Mittag-Leffler function which is generalization of various special functions [9]:

$$
E_{\alpha}(z)=\sum_{k=0}^{\infty} \frac{z^{n}}{\Gamma(n \alpha+1)}, \quad z, \alpha \in \mathbb{C}, \operatorname{Re}(\alpha)>0
$$

This function has been generalized by several mathematicians and utilized in various subjects of science and engineering. In [1] it is extended as follows.

Definition 7 Let $\mu, v, l, \gamma, d \in \mathbb{C}, \operatorname{Re}(\mu), \operatorname{Re}(v), \operatorname{Re}(l)>0, \operatorname{Re}(d)>\operatorname{Re}(\gamma)>0$ with $p \geq 0$, $\delta>0$ and $0<m \leq \delta+\operatorname{Re}(\mu)$. Then the extended generalized Mittag-Leffler function $E_{\mu, v, l}^{\gamma, \delta, m, d}(t ; p)$ is defined by

$$
E_{\mu, v, l}^{\gamma, \delta, m, d}(t ; p)=\sum_{i=0}^{\infty} \frac{\beta_{p}(\gamma+i m, d-\gamma)}{\beta(\gamma, d-\gamma)} \frac{(d)_{i m}}{\Gamma(\mu i+\nu)} \frac{t^{i}}{(l)_{i \delta}},
$$

where $\beta_{p}$ is the generalized Beta function defined by [1]

$$
\beta_{p}(u, v)=\int_{0}^{1} x^{u-1}(1-x)^{\nu-1} e^{\frac{-p}{x(1-x)}} d x
$$

where $(\cdot)_{i m}$ is the Pochhammer symbol defined by $(\cdot)_{i m}=\frac{\Gamma(\cdot+i m)}{\Gamma(\cdot)}$. 
The aim of this paper is to explore new Caputo $k$-fractional integral inequalities by using Grüss type variable bounds of functions having $n$-time derivatives absolutely continuous. Also, for Grüss type conditions on two $n$-time differentiable functions, more fractional integral inequalities are obtained. Further Chebyshev inequality (1.16) is used to produce some interesting consequences. Moreover, Caputo $k$-fractional differential equations involving the extended generalized Mittag-Leffler function are analyzed and their solutions are proposed via the Laplace transform technique.

\section{Caputo $k$-fractional inequalities}

The first result is derived for the function having $n$-time derivatives absolutely continuous and bounded by variable functions.

Theorem 2 Let $g, h_{1}, h_{2}:[a, b] \rightarrow \mathbb{R}$ be the functions such that $g, h_{1}, h_{2} \in A C^{n}[a, b]$. Also, let $\forall x \in[a, b]$

$$
h_{1}^{(n)}(x) \leq g^{(n)}(x) \leq h_{2}^{(n)}(x)
$$

Then the following inequality for the Caputo $k$-fractional derivatives holds:

$$
\begin{aligned}
& \left(C_{k, a+}^{\alpha} h_{2}\right)(x)\left(C_{k, a+}^{\beta} g\right)(x)+\left(C_{k, a+}^{\beta} h_{1}\right)(x)\left(C_{k, a+}^{\alpha} g\right)(x) \\
& \quad \geq\left(C_{k, a+}^{\alpha} h_{2}\right)(x)\left(C_{k, a+}^{\beta} h_{1}\right)(x)+\left(C_{k, a+}^{\alpha} g\right)(x)\left(C_{k, a+}^{\beta} g\right)(x) .
\end{aligned}
$$

Proof From (2.1), $\forall u, v \in[a, b]$, one can obtain

$$
\left(h_{2}^{(n)}(u)-g^{(n)}(u)\right)\left(g^{(n)}(v)-h_{1}^{(n)}(v)\right) \geq 0 .
$$

This inequality further takes the form

$$
h_{2}^{(n)}(u) g^{(n)}(v)+h_{1}^{(n)}(v) g^{(n)}(u) \geq h_{1}^{(n)}(v) h_{2}^{(n)}(u)+g^{(n)}(u) g^{(n)}(v) .
$$

Multiplying by $(x-u)^{n-\frac{\alpha}{k}-1}$ on both sides of above inequality and then integrating with respect to $u$ over $[a, x]$, we get

$$
\begin{aligned}
& \int_{a}^{x} h_{2}^{(n)}(u) g^{(n)}(v)(x-u)^{n-\frac{\alpha}{k}-1} d u+\int_{a}^{x} h_{1}^{(n)}(v) g^{(n)}(u)(x-u)^{n-\frac{\alpha}{k}-1} d u \\
& \quad \geq \int_{a}^{x} h_{1}^{(n)}(v) h_{2}^{(n)}(u)(x-u)^{n-\frac{\alpha}{k}-1} d u+\int_{a}^{x} g^{(n)}(u) g^{(n)}(v)(x-u)^{n-\frac{\alpha}{k}-1} d u .
\end{aligned}
$$

Using the definition of Caputo $k$-fractional derivatives, the following inequality is yielded:

$$
\begin{aligned}
& g^{(n)}(v) k \Gamma_{k}\left(n-\frac{\alpha}{k}\right)\left(C_{k, a+}^{\alpha} h_{2}\right)(x)+h_{1}^{(n)}(v) k \Gamma_{k}\left(n-\frac{\alpha}{k}\right)\left(C_{k, a+}^{\alpha} g\right)(x) \\
& \quad \geq h_{1}^{(n)}(v) k \Gamma_{k}\left(n-\frac{\alpha}{k}\right)\left(C_{k, a+}^{\alpha} h_{2}\right)(x)+f^{(n)}(v) k \Gamma_{k}\left(n-\frac{\alpha}{k}\right)\left(C_{k, a+}^{\alpha} g\right)(x) .
\end{aligned}
$$


Now multiplying the above inequality by $(x-v)^{n-\frac{\beta}{k}-1}$ on both sides and then integrating with respect to $v$ over $[a, x]$, we get

$$
\begin{aligned}
k \Gamma_{k}\left(n-\frac{\alpha}{k}\right)\left(C_{k, a+}^{\alpha} h_{2}\right)(x) \int_{a}^{x} g^{(n)}(v)(x-v)^{n-\frac{\beta}{k}-1} d v \\
\quad+k \Gamma_{k}\left(n-\frac{\alpha}{k}\right)\left(C_{k, a+a}^{\alpha} g\right)(x) \int_{a}^{x} h_{1}^{(n)}(v)(x-v)^{n-\frac{\beta}{k}-1} d v \\
\geq k \Gamma_{k}\left(n-\frac{\alpha}{k}\right)\left(C_{k, a+}^{\alpha} h_{2}\right)(x) \int_{a}^{x} h_{1}^{(n)}(v)(x-v)^{n-\frac{\beta}{k}-1} d v \\
+k \Gamma_{k}\left(n-\frac{\alpha}{k}\right)\left(C_{k, a+}^{\alpha} g\right)(x) \int_{a}^{x} g^{(n)}(v)(x-v)^{n-\frac{\beta}{k}-1} d v .
\end{aligned}
$$

Again, by using the definition of Caputo $k$-fractional derivatives, one can obtain (2.2).

Some special cases of Theorem 2 have been derived in the following results.

Corollary 1 If we take $k=1$ in Theorem 2, then the following inequality holds:

$$
\begin{aligned}
& \left(C_{a+}^{\alpha} h_{2}\right)(x)\left(C_{a+}^{\beta} g\right)(x)+\left(C_{a+}^{\beta} h_{1}\right)(x)\left(C_{a+}^{\alpha} g\right)(x) \\
& \quad \geq\left(C_{a+}^{\alpha} h_{2}\right)(x)\left(C_{a+}^{\beta} h_{1}\right)(x)+\left(C_{a+}^{\alpha} g\right)(x)\left(C_{a+}^{\beta} g\right)(x) .
\end{aligned}
$$

Corollary 2 If we take $\alpha=\beta$ in Theorem 2 , then the following inequality holds:

$$
\begin{aligned}
& \left(C_{k, a+}^{\alpha} g\right)(x)\left(\left(C_{k, a+}^{\alpha} h_{2}\right)(x)+\left(C_{k, a+}^{\alpha} h_{1}\right)(x)\right) \\
& \quad \geq\left(C_{k, a+}^{\alpha} h_{2}\right)(x)\left(C_{k, a+}^{\alpha} h_{1}\right)(x)+\left(\left(C_{k, a+}^{\alpha} g\right)(x)\right)^{2} .
\end{aligned}
$$

Corollary 3 Using (1.16) in (2.6), we obtain the following inequality:

$$
\begin{aligned}
& \frac{(x-a)^{n-\frac{\alpha}{k}}}{k \Gamma_{k}\left(n-\frac{\alpha}{k}+k\right)}\left[\left(C_{k, a+a}^{\alpha} g * h_{2}\right)(x)+\left(C_{k, a+}^{\alpha} g * h_{1}\right)(x)\right] \\
& \quad \geq\left(C_{k, a+a}^{\alpha} g\right)(x)\left(\left(C_{k, a+}^{\alpha} h_{2}\right)(x)+\left(C_{k, a+}^{\alpha} h_{1}\right)(x)\right) \\
& \quad \geq\left(C_{k, a+}^{\alpha} h_{2}\right)(x)\left(C_{k, a+}^{\alpha} h_{1}\right)(x)+\left(\left(C_{k, a+}^{\alpha} g\right)(x)\right)^{2} .
\end{aligned}
$$

Corollary 4 Let $g:[a, b] \rightarrow \mathbb{R}$ be a function such that $g \in A C^{n}[a, b]$. If $m, M \in \mathbb{R}$ satisfying $m \leq g^{(n)}(x) \leq M$, then $\forall x \in[a, b]$ the following inequality for the Caputo $k$-fractional derivatives holds:

$$
\begin{aligned}
& \frac{M(x-a)^{n-\frac{\alpha}{k}}}{k \Gamma_{k}\left(n-\frac{\alpha}{k}+k\right)}\left(C_{k, a+}^{\beta} g\right)(x)+\frac{m(x-a)^{n-\frac{\beta}{k}}}{k \Gamma_{k}\left(n-\frac{\beta}{k}+k\right)}\left(C_{k, a+a}^{\alpha} g\right)(x) \\
& \quad \geq \frac{m M(x-a)^{2 n-\frac{\alpha+\beta}{k}}}{k^{2} \Gamma_{k}\left(n-\frac{\alpha}{k}+k\right) \Gamma_{k}\left(n-\frac{\beta}{k}+k\right)}+\left(C_{k, a+a}^{\alpha} g\right)(x)\left(C_{k, a+}^{\beta} g\right)(x) .
\end{aligned}
$$

Proof If we take $h_{1}=\frac{m x^{n}}{n !}$ and $h_{2}=\frac{M x^{n}}{n !}$ in Theorem 2 and follow the rest of the proof along the same lines as the proof of Theorem 2, inequality (2.8) can be obtained. 
Corollary 5 If we take $\alpha=\beta$ in Corollary 4 , we obtain the following inequality:

$$
\begin{aligned}
& \frac{(m+M)(x-a)^{n-\frac{\alpha}{k}}}{k \Gamma_{k}\left(n-\frac{\alpha}{k}+k\right)}\left(C_{k, a+}^{\alpha} g\right)(x)-\left(\left(C_{k, a+}^{\alpha} g\right)(x)\right)^{2} \\
& \quad \geq \frac{m M(x-a)^{2 n-\frac{2 \alpha}{k}}}{\left(k \Gamma_{k}\left(n-\frac{\alpha}{k}+k\right)\right)^{2}} .
\end{aligned}
$$

Corollary 6 If we take $k=1$ in Corollary 4, then the following inequality holds:

$$
\begin{aligned}
& \frac{M(x-a)^{n-\alpha}}{\Gamma(n-\alpha+1)}\left(C_{a+}^{\beta} g\right)(x)+\frac{m(x-a)^{n-\beta}}{\Gamma(n-\beta+1)}\left(C_{a+}^{\alpha} g\right)(x)-\left(C_{a+}^{\alpha} g\right)(x)\left(C_{a+g}^{\beta} g\right)(x) \\
& \quad \geq \frac{m M(x-a)^{2 n-\alpha-\beta}}{\Gamma(n-\alpha+1) \Gamma(n-\beta+1)} .
\end{aligned}
$$

Corollary 7 If we take $\alpha=\beta$ in Corollary 6, then the following inequality holds:

$$
\begin{aligned}
(m+M) & \frac{(x-a)^{n-\alpha}}{\Gamma(n-\alpha+1)}\left(C_{a+}^{\alpha} g\right)(x)-\left(\left(C_{a+d}^{\alpha} g\right)(x)\right)^{2} \\
\geq & \frac{m M(x-a)^{2 n-2 \alpha}}{(\Gamma(n-\alpha+1))^{2}} .
\end{aligned}
$$

The next result is derived for two functions $f$ and $g$ having $n$th derivatives absolutely continuous and bounded by the variable functions.

Theorem 3 Let $f, g, h_{1}, h_{2}, t_{1}, t_{2}:[a, b] \rightarrow \mathbb{R}$ be the functions such that $f, g, h_{1}, h_{2}, t_{1}, t_{2} \in$ $A C^{n}[a, b]$. Also, let $\forall x \in[a, b]$

$$
h_{1}^{(n)}(x) \leq g^{(n)}(x) \leq h_{2}^{(n)}(x)
$$

and

$$
t_{1}^{(n)}(x) \leq f^{(n)}(x) \leq t_{2}^{(n)}(x)
$$

Then the following inequalities for the Caputo k-fractional derivatives hold:

$$
\begin{aligned}
& \left(C_{k, a+}^{\alpha} h_{2}\right)(x)\left(C_{k, a+}^{\beta} f\right)(x)+\left(C_{k, a+}^{\beta} t_{1}\right)(x)\left(C_{k, a+a}^{\alpha} g\right)(x) \\
& \geq\left(C_{k, a+}^{\alpha} h_{2}\right)(x)\left(C_{k, a+}^{\beta} t_{1}\right)(x)+\left(C_{k, a+}^{\alpha} g\right)(x)\left(C_{k, a+}^{\beta} f\right)(x), \\
& \left(C_{k, a+}^{\beta} t_{2}\right)(x)\left(C_{k, a+}^{\alpha} g\right)(x)+\left(C_{k, a+}^{\alpha} h_{1}\right)(x)\left(C_{k, a+}^{\beta} g\right)(x) \\
& \geq\left(C_{k, a+}^{\alpha} h_{1}\right)(x)\left(C_{k, a+}^{\beta} t_{2}\right)(x)+\left(C_{k, a+}^{\alpha} g\right)(x)\left(C_{k, a+}^{\beta} f\right)(x), \\
& \left(C_{k, a+}^{\alpha} h_{2}\right)(x)\left(C_{k, a+}^{\beta} t_{2}\right)(x)+\left(C_{k, a+}^{\alpha} g\right)(x)\left(C_{k, a+}^{\beta} f\right)(x) \\
& \geq\left(C_{k, a+}^{\alpha} h_{2}\right)(x)\left(C_{k, a+}^{\beta} f\right)(x)+\left(C_{k, a+}^{\beta} t_{2}\right)(x)\left(C_{k, a+}^{\alpha} g\right)(x), \\
& \left(C_{k, a+}^{\alpha} h_{1}\right)(x)\left(C_{k, a+}^{\beta} t_{1}\right)(x)+\left(C_{k, a+}^{\alpha} g\right)(x)\left(C_{k, a+}^{\beta} f\right)(x) \\
& \geq\left(C_{k, a+}^{\alpha} h_{1}\right)(x)\left(C_{k, a+}^{\beta} g\right)(x)+\left(C_{k, a+}^{\beta} t_{1}\right)(x)\left(C_{k, a+}^{\alpha} g\right)(x) .
\end{aligned}
$$


Proof Using (2.12) and (2.13), the following inequality holds:

$$
\left(h_{2}^{(n)}(u)-g^{(n)}(u)\right)\left(f^{(n)}(v)-t_{1}^{(n)}(v)\right) \geq 0 .
$$

This further takes the form

$$
h_{2}^{(n)}(u) f^{(n)}(v)+t_{1}^{(n)}(v) g^{(n)}(u) \geq h_{2}^{(n)}(u) t_{1}^{(n)}(v)+g^{(n)}(u) f^{(n)}(v) .
$$

Multiplying by $(x-u)^{n-\frac{\alpha}{k}-1}$ on both sides of the above inequality and then integrating with respect to $u$ over $[a, x]$, we get

$$
\begin{aligned}
& \int_{a}^{x} h_{2}^{(n)}(u) f^{(n)}(v)(x-u)^{n-\frac{\alpha}{k}-1} d u+\int_{a}^{x} t_{1}^{(n)}(v) g^{(n)}(u)(x-u)^{n-\frac{\alpha}{k}-1} d u \\
& \quad \geq \int_{a}^{x} h_{2}^{(n)}(u) t_{1}^{(n)}(v)(x-u)^{n-\frac{\alpha}{k}-1} d u+\int_{a}^{x} g^{(n)}(u) f^{(n)}(v)(x-u)^{n-\frac{\alpha}{k}-1} d u .
\end{aligned}
$$

Using the definition of Caputo $k$-fractional derivatives, we obtain

$$
\begin{aligned}
& f^{(n)}(v) k \Gamma_{k}\left(n-\frac{\alpha}{k}\right)\left(C_{k, a+}^{\alpha} h_{2}\right)(x)+t_{1}^{(n)}(v) k \Gamma_{k}\left(n-\frac{\alpha}{k}\right)\left(C_{k, a+}^{\alpha} g\right)(x) \\
& \quad \geq t_{1}^{(n)}(v) k \Gamma_{k}\left(n-\frac{\alpha}{k}\right)\left(C_{k, a+}^{\alpha} h_{2}\right)(x)+f^{(n)}(v) k \Gamma_{k}\left(n-\frac{\alpha}{k}\right)\left(C_{k, a+}^{\alpha} g\right)(x) .
\end{aligned}
$$

Now, multiplying by $(x-v)^{n-\frac{\beta}{k}-1}$ on both sides of the above inequality and then integrating with respect to $v$ over $[a, x]$, we get

$$
\begin{aligned}
k \Gamma_{k}\left(n-\frac{\alpha}{k}\right)\left(C_{k, a+}^{\alpha} h_{2}\right)(x) \int_{a}^{x} f^{(n)}(v)(x-v)^{n-\frac{\beta}{k}-1} d v \\
\quad+k \Gamma_{k}\left(n-\frac{\alpha}{k}\right)\left(C_{k, a+a}^{\alpha} g\right)(x) \int_{a}^{x} t_{1}^{(n)}(v)(x-v)^{n-\frac{\beta}{k}-1} d v \\
\geq k \Gamma_{k}\left(n-\frac{\alpha}{k}\right)\left(C_{k, a+}^{\alpha} h_{2}\right)(x) \int_{a}^{x} t_{1}^{(n)}(v)(x-v)^{n-\frac{\beta}{k}-1} d v \\
\quad+k \Gamma_{k}\left(n-\frac{\alpha}{k}\right)\left(C_{k, a+a}^{\alpha} g\right)(x) \int_{a}^{x} f^{(n)}(v)(x-v)^{n-\frac{\beta}{k}-1} d v .
\end{aligned}
$$

Again using the definition of Caputo $k$-fractional derivatives, inequality (2.14) can be obtained.

For the proof of (2.15), (2.16), and (2.17), the following inequalities can be used respectively:

$$
\begin{aligned}
& \left(g^{(n)}(u)-h_{1}^{(n)}(u)\right)\left(t_{2}^{(n)}(v)-f^{(n)}(v)\right) \geq 0, \\
& \left(h_{2}^{(n)}(u)-g^{(n)}(u)\right)\left(f^{(n)}(v)-t_{2}^{(n)}(v)\right) \leq 0, \\
& \left(h_{1}^{(n)}(u)-g^{(n)}(u)\right)\left(f^{(n)}(v)-t_{1}^{(n)}(v)\right) \leq 0 .
\end{aligned}
$$

The rest of the proof follows along the same lines as the proof of (2.14).

The further consequences of Theorem 3 are stated in the following corollaries. 
Corollary 8 If we take $\alpha=\beta$ in Theorem 3 , then the following results hold:

$$
\begin{aligned}
& \left(C_{k, a+}^{\alpha} h_{2}\right)(x)\left(C_{k, a+}^{\alpha} f\right)(x)+\left(C_{k, a+}^{\alpha} t_{1}\right)(x)\left(C_{k, a+}^{\alpha} g\right)(x) \\
& \geq\left(C_{k, a+}^{\alpha} h_{2}\right)(x)\left(C_{k, a+}^{\alpha} t_{1}\right)(x)+\left(C_{k, a+}^{\alpha} f\right)(x)\left(C_{k, a+}^{\alpha} g\right)(x), \\
& \left(C_{k, a+}^{\alpha} t_{2}\right)(x)\left(C_{k, a+}^{\alpha} g\right)(x)+\left(C_{k, a+}^{\alpha} h_{1}\right)(x)\left(C_{k, a+}^{\alpha} f\right)(x) \\
& \quad \geq\left(C_{k, a+}^{\alpha} h_{1}\right)(x)\left(C_{k, a+}^{\alpha} t_{2}\right)(x)+\left(C_{k, a+}^{\alpha} g\right)(x)\left(C_{k, a+}^{\alpha} f\right)(x), \\
& \left(C_{k, a+}^{\alpha} h_{2}\right)(x)\left(C_{k, a+}^{\alpha} t_{2}\right)(x)+\left(C_{k, a+}^{\alpha} f\right)(x)\left(C_{k, a+a}^{\alpha} g\right)(x) \\
& \geq\left(C_{k, a+}^{\alpha} h_{2}\right)(x)\left(C_{k, a+}^{\alpha} f\right)(x)+\left(C_{k, a+}^{\alpha} t_{2}\right)(x)\left(C_{k, a+}^{\alpha} g\right)(x), \\
& \left(C_{k, a+}^{\alpha} h_{1}\right)(x)\left(C_{k, a+}^{\alpha} t_{1}\right)(x)+\left(C_{k, a+}^{\alpha} f\right)(x)\left(C_{k, a+}^{\alpha} g\right)(x) \\
& \geq\left(C_{k, a+}^{\alpha} h_{1}\right)(x)\left(C_{k, a+}^{\alpha} f\right)(x)+\left(C_{k, a+}^{\alpha} t_{1}\right)(x)\left(C_{k, a+}^{\alpha} g\right)(x) .
\end{aligned}
$$

Corollary 9 Using Theorem 1 in Corollary 8, the following results hold:

$$
\begin{aligned}
& \frac{(x-a)^{n-\frac{\alpha}{k}}}{k \Gamma_{k}\left(n-\frac{\alpha}{k}+k\right)}\left[\left(C_{k, a+}^{\alpha} h_{2} * f\right)(x)+\left(C_{k, a+}^{\alpha} g * t_{1}\right)(x)\right] \\
& \quad \geq\left(C_{k, a+}^{\alpha} h_{2}\right)(x)\left(C_{k, a+}^{\alpha} f\right)(x)+\left(C_{k, a+}^{\alpha} t_{1}\right)(x)\left(C_{k, a+}^{\alpha} g\right)(x) \\
& \quad \geq\left(C_{k, a+}^{\alpha} h_{2}\right)(x)\left(C_{k, a+}^{\alpha} t_{1}\right)(x)+\left(C_{k, a+}^{\alpha} f\right)(x)\left(C_{k, a+}^{\alpha} g\right)(x), \\
& \frac{(x-a)^{n-\frac{\alpha}{k}}}{k \Gamma_{k}\left(n-\frac{\alpha}{k}+k\right)}\left[\left(C_{k, a+}^{\alpha} g * t_{2}\right)(x)+\left(C_{k, a+}^{\alpha} f * h_{1}\right)(x)\right] \\
& \quad \geq\left(C_{k, a+}^{\alpha} t_{2}\right)(x)\left(C_{k, a+}^{\alpha} g\right)(x)+\left(C_{k, a+}^{\alpha} h_{1}\right)(x)\left(C_{k, a+}^{\alpha} f\right)(x) \\
& \quad \geq\left(C_{k, a+}^{\alpha} h_{1}\right)(x)\left(C_{k, a+}^{\alpha} t_{2}\right)(x)+\left(C_{k, a+}^{\alpha} f\right)(x)\left(C_{k, a+}^{\alpha} g\right)(x), \\
& \frac{(x-a)^{n-\frac{\alpha}{k}}}{k \Gamma_{k}\left(n-\frac{\alpha}{k}+k\right)}\left[\left(C_{k, a+}^{\alpha} h_{2} * t_{2}\right)(x)+\left(C_{k, a+}^{\alpha} f * g\right)(x)\right] \\
& \quad \geq\left(C_{k, a+}^{\alpha} h_{2}\right)(x)\left(C_{k, a+}^{\alpha} t_{2}\right)(x)+\left(C_{k, a+}^{\alpha} f\right)(x)\left(C_{k, a+}^{\alpha} g\right)(x) \\
& \quad \geq\left(C_{k, a+}^{\alpha} h_{2}\right)(x)\left(C_{k, a+}^{\alpha} f\right)(x)+\left(C_{k, a+}^{\alpha} t_{2}\right)(x)\left(C_{k, a+}^{\alpha} g\right)(x), \\
& \frac{(x-a)^{n-\frac{\alpha}{k}}}{k \Gamma_{k}\left(n-\frac{\alpha}{k}+k\right)}\left[\left(C_{k, a+}^{\alpha} h_{1} * t_{1}\right)(x)+\left(C_{k, a+}^{\alpha} f * g\right)(x)\right] \\
& \quad \geq\left(C_{k, a+}^{\alpha} h_{1}\right)(x)\left(C_{k, a+}^{\alpha} t_{1}\right)(x)+\left(C_{k, a+}^{\alpha} f\right)(x)\left(C_{k, a+}^{\alpha} g\right)(x) \\
& \quad \geq\left(C_{k, a+}^{\alpha} h_{1}\right)(x)\left(C_{k, a+}^{\alpha} f\right)(x)+\left(C_{k, a+}^{\alpha} t_{1}\right)(x)\left(C_{k, a+}^{\alpha} f\right)(x) .
\end{aligned}
$$

Corollary 10 If we take $k=1$ in Theorem 3, then the following inequalities hold:

$$
\begin{aligned}
& \left(C_{a+}^{\alpha} h_{2}\right)(x)\left(C_{a+}^{\beta} f\right)(x)+\left(C_{a+}^{\beta} t_{1}\right)(x)\left(C_{a+}^{\alpha} g\right)(x) \\
& \geq\left(C_{a+}^{\alpha} h_{2}\right)(x)\left(C_{a+}^{\beta} t_{1}\right)(x)+\left(C_{a+}^{\alpha} g\right)(x)\left(C_{a+}^{\beta} f\right)(x), \\
& \left(C_{a+}^{\beta} t_{2}\right)(x)\left(C_{a+}^{\alpha} g\right)(x)+\left(C_{a+}^{\alpha} h_{1}\right)(x)\left(C_{a+}^{\beta} f\right)(x) \\
& \quad \geq\left(C_{a+}^{\alpha} h_{1}\right)(x)\left(C_{a+}^{\beta} t_{2}\right)(x)+\left(C_{a+}^{\alpha} g\right)(x)\left(C_{a+}^{\beta} f\right)(x),
\end{aligned}
$$




$$
\begin{aligned}
& \left(C_{a+}^{\alpha} h_{2}\right)(x)\left(C_{a+}^{\beta} t_{2}\right)(x)+\left(C_{a+}^{\alpha} g\right)(x)\left(C_{a+}^{\beta} f\right)(x) \\
& \geq\left(C_{a+}^{\alpha} h_{2}\right)(x)\left(C_{a+}^{\beta} f\right)(x)+\left(C_{a+}^{\beta} t_{2}\right)(x)\left(C_{a+}^{\alpha} g\right)(x), \\
& \left(C_{a+}^{\alpha} h_{1}\right)(x)\left(C_{a+}^{\beta} t_{1}\right)(x)+\left(C_{a+}^{\alpha} g\right)(x)\left(C_{a+}^{\beta} f\right)(x) \\
& \quad \geq\left(C_{a+}^{\alpha} h_{1}\right)(x)\left(C_{a+}^{\beta} f\right)(x)+\left(C_{a+}^{\beta} t_{1}\right)(x)\left(C_{a+}^{\alpha} g\right)(x) .
\end{aligned}
$$

Corollary 11 Let $f, g:[a, b] \rightarrow \mathbb{R}$ be the functions such thatf, $g \in A C^{n}[a, b]$. If $m, M, n, N \in$ $\mathbb{R}$ satisfy $m \leq g^{(n)}(x) \leq M$ and $l \leq f^{(n)}(x) \leq L$, then $\forall x \in[a, b]$ the following inequalities for the Caputo k-fractional derivatives hold:

$$
\begin{aligned}
& \frac{M(x-a)^{n-\frac{\alpha}{k}}}{k \Gamma_{k}\left(n-\frac{\alpha}{k}+k\right)}\left(C_{k, a+}^{\beta} f\right)(x)+\frac{l(x-a)^{n-\frac{\beta}{k}}}{k \Gamma_{k}\left(n-\frac{\beta}{k}+k\right)}\left(C_{k, a+a}^{\alpha} g\right)(x) \\
& \geq \frac{M l(x-a)^{2 n-\frac{\alpha+\beta}{k}}}{k \Gamma_{k}\left(n-\frac{\alpha}{k}+k\right) k \Gamma_{k}\left(n-\frac{\beta}{k}+k\right)}+\left(C_{k, a+a}^{\alpha} g\right)(x)\left(C_{k, a+}^{\beta} f\right)(x), \\
& \frac{L(x-a)^{n-\frac{\beta}{k}}}{k \Gamma_{k}\left(n-\frac{\beta}{k}+k\right)}\left(C_{k, a+a}^{\alpha} g\right)(x)+\frac{m(x-a)^{n-\frac{\alpha}{k}}}{k \Gamma_{k}\left(n-\frac{\alpha}{k}+k\right)}\left(C_{k, a+}^{\beta} f\right)(x) \\
& \geq \frac{m L(x-a)^{2 n-\frac{\alpha+\beta}{k}}}{k \Gamma_{k}\left(n-\frac{\alpha}{k}+k\right) k \Gamma_{k}\left(n-\frac{\beta}{k}+k\right)}+\left(C_{k, a+}^{\alpha} g\right)(x)\left(C_{k, a+}^{\beta} f\right)(x), \\
& \frac{M L(x-a)^{2 n-\frac{\alpha+\beta}{k}}}{k \Gamma_{k}\left(n-\frac{\alpha}{k}+k\right) k \Gamma_{k}\left(n-\frac{\beta}{k}+k\right)}+\left(C_{k, a+}^{\alpha} g\right)(x)\left(C_{k, a}^{\beta} f\right)(x) \\
& \geq \frac{M(x-a)^{n-\frac{\alpha}{k}}}{k \Gamma_{k}\left(n-\frac{\alpha}{k}+k\right)}\left(C_{k, a+}^{\beta} f\right)(x)+\frac{L(x-a)^{n-\frac{\beta}{k}}}{k \Gamma_{k}\left(n-\frac{\beta}{k}+k\right)}\left(C_{k, a+}^{\alpha} g\right)(x), \\
& \frac{m l(x-a)^{2 n-\frac{\alpha+\beta}{k}}}{k \Gamma_{k}\left(n-\frac{\alpha}{k}+k\right) k \Gamma_{k}\left(n-\frac{\beta}{k}+k\right)}+\left(C_{k, a+a}^{\alpha} g\right)(x)\left(C_{k, a+}^{\beta} f\right)(x) \\
& \geq \frac{m(x-a)^{n-\frac{\alpha}{k}}}{k \Gamma_{k}\left(n-\frac{\alpha}{k}+k\right)}\left(C_{k, a+}^{\beta} f\right)(x)+l \frac{(x-a)^{n-\frac{\beta}{k}}}{k \Gamma_{k}\left(n-\frac{\beta}{k}+k\right)}\left(C_{k, a+a}^{\alpha} g\right)(x) .
\end{aligned}
$$

Proof If we take $h_{1}=\frac{m x^{n}}{n !}, h_{2}=\frac{M x^{n}}{n !}, t_{1}=\frac{l x^{n}}{n !}$, and $t_{2}=\frac{L x^{n}}{n !}$ in Theorem 3 and follow the rest of the proof along the same lines as the proof of Theorem 3 , the required inequality can be obtained.

Corollary 12 If we take $k=1$ in Corollary 11, then the following inequalities hold:

$$
\begin{aligned}
& \frac{M(x-a)^{n-\alpha}}{\Gamma(n-\alpha+1)}\left(C_{a+}^{\beta} f\right)(x)+l \frac{(x-a)^{n-\beta}}{\Gamma(n-\beta+1)}\left(C_{a+}^{\alpha} g\right)(x) \\
& \quad \geq \frac{M l(x-a)^{2 n-\alpha-\beta}}{\Gamma(n-\alpha+1) \Gamma(n-\beta+1)}+\left(C_{a+}^{\alpha} g\right)(x)\left(C_{a+}^{\beta} f\right)(x), \\
& \frac{L(x-a)^{n-\beta}}{\Gamma(n-\beta+1)}\left(C_{a+}^{\alpha} g\right)(x)+\frac{m(x-a)^{n-\alpha}}{\Gamma(n-\alpha+1)}\left(C_{a+}^{\beta} f\right)(x) \\
& \quad \geq \frac{m L(x-a)^{2 n-\alpha-\beta}}{\Gamma(n-\alpha+1) \Gamma(n-\beta+1)}+\left(C_{a+}^{\alpha} g\right)(x)\left(C_{a+}^{\beta} f\right)(x),
\end{aligned}
$$




$$
\begin{aligned}
& \frac{M L(x-a)^{2 n-\alpha-\beta}}{\Gamma(n-\alpha+1) \Gamma(n-\beta+1)}+\left(C_{a+}^{\alpha} g\right)(x)\left(C_{a+}^{\beta} f\right)(x) \\
& \geq \frac{M(x-a)^{n-\alpha}}{\Gamma(n-\alpha+1)}\left(C_{a+}^{\beta} f\right)(x)+L \frac{(x-a)^{n-\beta}}{\Gamma(n-\beta+1)}\left(C_{a+}^{\alpha} g\right)(x), \\
& \frac{m l(x-a)^{2 n-\alpha-\beta}}{\Gamma(n-\alpha+1) \Gamma(n-\beta+1)}+\left(C_{a+}^{\alpha} g\right)(x)\left(C_{a+}^{\beta} f\right)(x) \\
& \quad \geq \frac{m(x-a)^{n-\alpha}}{\Gamma(n-\alpha+1)}\left(C_{a+}^{\beta} f\right)(x)+\frac{l(x-a)^{n-\beta}}{\Gamma(n-\beta+1)}\left(C_{a+}^{\alpha} g\right)(x) .
\end{aligned}
$$

\section{Fractional differential equations}

In this section, we formulate some generalized fractional differential equations and also obtain their solutions. First we find the Laplace transform of Caputo $k$-fractional derivative.

Lemma 2 Let $\alpha>0, k \geq 1$, and $\alpha \notin\{1,2,3, \ldots\}, n=[\alpha]+1$. Then the Laplace transform of left-sided Caputo $k$-fractional derivative is given by

$$
\mathcal{L}\left\{\left(C_{k, 0+1}^{\alpha} f\right)(x)\right\}=\frac{\Gamma_{k}(n k-\alpha)}{(k s)^{n-\frac{\alpha}{k}} \Gamma_{k}\left(n-\frac{\alpha}{k}\right)}\left[s^{n} \mathcal{L}\{f(t)\}-\sum_{i=0}^{n-1} s^{n-i-1} f^{(i)}\left(0^{+}\right)\right] .
$$

Proof For $a=0$, the left-sided Caputo $k$-fractional derivative is given by

$$
\left(C_{k, 0+}^{\alpha} f\right)(x)=\frac{1}{k \Gamma_{k}\left(n-\frac{\alpha}{k}\right)} \int_{0}^{x} \frac{f^{(n)}(t)}{(x-t)^{\frac{\alpha}{k}-n+1}} d t .
$$

The Laplace convolution operator of two functions $f(t)$ and $g(t)$ given on $\mathbb{R}^{+}$is defined by the integral

$$
f * g=(f * g)(x)=\int_{0}^{x} f(x-t) g(t) d t
$$

which has the commutative property, i.e., $f * g=g * f$.

Therefore (3.2) can be written as $\left(f^{(n)} * g\right)(x)$, where $f(t)=t^{n-\frac{\alpha}{k}-1}$ and $g(t)=f^{(n)}(t)$

$$
\left(C_{k, 0+}^{\alpha} f\right)(x)=\frac{1}{k \Gamma_{k}\left(n-\frac{\alpha}{k}\right)} f^{(n)} *(t)^{n-\frac{\alpha}{k}-1} .
$$

Now, taking Laplace transform and using the property $\mathcal{L}\{f * g\}=\mathcal{L}\{f\} \mathcal{L}\{g\}$ of Laplace transform, we have

$$
\mathcal{L}\left\{\left(C_{k, 0+0}^{\alpha} f\right)(x)\right\}=\frac{1}{k \Gamma_{k}\left(n-\frac{\alpha}{k}\right)} \mathcal{L}\left\{f^{(n)}(t)\right\} \mathcal{L}\left\{(t)^{n-\frac{\alpha}{k}-1}\right\} .
$$

Using formulas $\mathcal{L}\left\{f^{(n)}(t)\right\}(s)=s^{n} \mathcal{L}\{f(t)\}-\sum_{i=0}^{n-1} s^{n-i-1} f^{(i)}\left(0^{+}\right)$and $\mathcal{L}\left\{t^{\alpha}\right\}(s)=\frac{\Gamma(\alpha+1)}{s^{\alpha+1}}$ of the Laplace transform, we get

$$
\mathcal{L}\left\{\left(C_{k, 0+}^{\alpha} f\right)(x)\right\}=\frac{\Gamma\left(n-\frac{\alpha}{k}\right)}{k \Gamma_{k}\left(n-\frac{\alpha}{k}\right) s^{n-\frac{\alpha}{k}}}\left[s^{n} \mathcal{L}\{f(t)\}-\sum_{i=0}^{n-1} s^{n-i-1} f^{(i)}\left(0^{+}\right)\right] .
$$

Using the property of gamma function $\Gamma\left(\frac{\alpha}{k}\right)=\frac{\Gamma_{k}(\alpha)}{k^{\frac{\alpha}{k}}-1}$, (3.1) can be obtained. 
Theorem 4 Let $a \in \mathbb{R}^{+}, \mu, v, l, \gamma, d \in \mathbb{C}, \operatorname{Re}(\mu), \operatorname{Re}(v), \operatorname{Re}(l)>0, \operatorname{Re}(d)>\operatorname{Re}(\gamma)>0$ with $p \geq 0, \delta>0$, and $0<m \leq \delta+\operatorname{Re}(\mu)$. Then, for $x>a$, the following results hold:

$$
\begin{aligned}
& C_{k, a+}^{\alpha}\left[(t-a)^{\nu-1} E_{\mu, \nu, l}^{\gamma, \delta, m, d}\left(w(t-a)^{\mu} ; p\right)\right](x) \\
& \quad=\frac{(x-a)^{\nu-\frac{\alpha}{k}-1} \Gamma_{k}(\mu i k+\nu k-n k) \Gamma_{k}(n k-\alpha)}{\Gamma_{k}\left(n-\frac{\alpha}{k}\right) \Gamma_{k}(\mu i k+\nu k-\alpha)} E_{\mu, v-n, l}^{\gamma, \delta, m, d}\left(w(t-a)^{\mu} ; p\right)
\end{aligned}
$$

and

$$
R_{k, a}^{\alpha}\left[(t-a)^{\nu-1} E_{\mu, \nu, l}^{\gamma, \delta, m, d}\left(w(t-a)^{\mu} ; p\right)\right](x)=\frac{(x-a)^{\nu-\frac{\alpha}{k}-1}}{k^{\frac{\alpha}{k}}} E_{\mu, \nu+\frac{\alpha}{k}, l}^{\gamma, \delta, m, d}\left(w(t-a)^{\mu} ; p\right) .
$$

Proof Using the definition of Mittag-Leffler function, we can write the left-hand side of equation (3.3) as follows:

$$
\begin{aligned}
& C_{k, a+}^{\alpha}\left[(t-a)^{\nu-1} E_{\mu, v, l}^{\gamma, \delta, m, d}\left(w(t-a)^{\mu} ; p\right)\right](x) \\
& \left.\quad=\sum_{i=0}^{\infty} \frac{\beta_{p}(\gamma+i m, d-\gamma)}{\beta(\gamma, d-\gamma)} \frac{(d)_{i m}}{\Gamma(\mu i+\nu)} \frac{w^{i}}{(l)_{i \delta}} C_{k, a+}^{\alpha}\left[(t-a)^{\mu i+v-1}\right)\right](x) .
\end{aligned}
$$

Using Lemma 1, we can write (3.5) as follows:

$$
\begin{aligned}
C_{k, a+}^{\alpha} & {\left[(t-a)^{v-1} E_{\mu, v, l}^{\gamma, \delta, m, d}\left(w(t-a)^{\mu} ; p\right)\right](x) } \\
= & \sum_{i=0}^{\infty}\left[\frac{\beta_{p}(\gamma+i m, d-\gamma)}{\beta(\gamma, d-\gamma)} \frac{(d)_{i m}}{\Gamma(\mu i+v)} \frac{w^{i}}{(l)_{i \delta}}\right. \\
& \left.\times \frac{(x-a)^{\mu i+v-\frac{\alpha}{k}-1} \Gamma(\mu i+v) \Gamma_{k}(\mu i k+v k-n k) \Gamma_{k}(n k-\alpha)}{\Gamma(\mu i+v-n) \Gamma_{k}\left(n-\frac{\alpha}{k}\right) \Gamma_{k}(\mu i k+\nu k-\alpha)}\right] .
\end{aligned}
$$

Using the definition of Mittag-Leffler function, (3.3) can be obtained.

Now consider the left-hand side of equation (3.4), using the definition of Mittag-Leffler function, we have

$$
\begin{aligned}
R_{k, a}^{\alpha} & {\left[(t-a)^{\nu-1} E_{\mu, \nu, l}^{\gamma, \delta, m, d}\left(w(t-a)^{\mu} ; p\right)\right](x) } \\
& =\sum_{i=0}^{\infty} \frac{\beta_{p}(\gamma+i m, d-\gamma)}{\beta(\gamma, d-\gamma)} \frac{(d)_{i m}}{\Gamma(\mu i+\nu)} \frac{w^{i}}{(l)_{i \delta}} R_{k, a}^{\alpha}\left[(t-a)^{\mu i+\nu-1}\right](x) .
\end{aligned}
$$

Using $R_{k, a}^{\alpha}(x-a)^{p}=\frac{(x-a)^{p-\frac{\alpha}{k}} \Gamma_{k}(p k+k)}{\Gamma_{k}(p k+k+\alpha)}$, we get

$$
\begin{aligned}
R_{k, a}^{\alpha} & {\left[(t-a)^{\nu-1} E_{\mu, v, l}^{\gamma, \delta, m, d}\left(w(t-a)^{\mu} ; p\right)\right](x) } \\
& =\sum_{i=0}^{\infty}\left[\frac{\beta_{p}(\gamma+i m, d-\gamma)}{\beta(\gamma, d-\gamma)} \frac{(d)_{i m}}{\Gamma(\mu i+v)} \frac{w^{i}}{\left(l_{i \delta}\right.} \frac{(x-a)^{\mu i+\nu-\frac{\alpha}{k}-1} \Gamma_{k}(k(\mu i+v))}{\Gamma_{k}(k(\mu i+\nu)+\alpha)}\right] .
\end{aligned}
$$


Using $\Gamma_{k}(\alpha)=k^{\frac{\alpha}{k}-1} \Gamma\left(\frac{\alpha}{k}\right)$, we have

$$
\begin{aligned}
R_{k, a}^{\alpha} & {\left[(t-a)^{\nu-1} E_{\mu, v, l}^{\gamma, \delta, m, d}\left(w(t-a)^{\mu} ; p\right)\right](x) } \\
\quad= & \sum_{i=0}^{\infty} \frac{\beta_{p}(\gamma+i m, d-\gamma)}{\beta(\gamma, d-\gamma)} \frac{(d)_{i m}}{\Gamma\left(\mu i+v+\frac{\alpha}{k}\right)} \frac{w^{i}}{(l)_{i \delta}} \frac{(x-a)^{\mu i+v-1-\frac{\alpha}{k}}}{k^{\frac{\alpha}{k}}}
\end{aligned}
$$

which gives the required result (3.4).

Theorem 5 Let $a \in \mathbb{R}^{+}, \mu, v, l, \gamma, d \in \mathbb{C}, \operatorname{Re}(\mu), \operatorname{Re}(v), \operatorname{Re}(l)>0, \operatorname{Re}(d)>\operatorname{Re}(\gamma)>0$ with $p \geq 0, \delta>0$ and $0<m \leq \delta+\operatorname{Re}(\mu)$. The differential equation

$$
\left(C_{k, 0+}^{\alpha} y\right)(x)=\lambda x^{\nu} E_{\mu, v+1, l}^{\gamma, \delta, m, d}\left(w x^{\mu} ; p\right)+f(x)
$$

with the initial condition $y\left(0^{+}\right)=c$, where $c$ is an arbitrary constant, has the solution as follows:

$$
\begin{aligned}
y(x)= & c_{1} c+\frac{\lambda k^{1-\alpha} \Gamma_{k}\left(1-\frac{\alpha}{k}\right)}{\Gamma_{k}(k-\alpha)} \sum_{i=0}^{\infty}\left[\frac{\beta_{p}(\gamma+i m, d-\gamma)}{\beta(\gamma, d-\gamma)} \frac{(d)_{i m}}{\Gamma(\mu i+\alpha+\nu+1)} \frac{w^{i} x^{\mu i+\alpha+\nu}}{(l)_{i \delta}}\right] \\
& +\frac{k^{1-\alpha} \Gamma_{k}\left(1-\frac{\alpha}{k}\right)}{\Gamma_{k}(k-\alpha)} R_{0^{+}}^{\alpha} f(x) .
\end{aligned}
$$

Proof Applying the Laplace transform to equation (3.6), we have

$$
\mathcal{L}\left\{\left(C_{k, 0+}^{\alpha} y\right)(x)\right\}(s)=\lambda \mathcal{L}\left\{x^{\nu} E_{\mu, v+1, l}^{\gamma, \delta, m, d}\left(w x^{\mu} ; p\right)\right\}(s)+\mathcal{L}\{f(x)\}
$$

Using Lemma 2 for $n=1$ and the definition of Mittag-Leffler function, we have

$$
\begin{aligned}
& \frac{\Gamma_{k}(k-\alpha)}{(k s)^{1-\alpha} \Gamma_{k}\left(1-\frac{\alpha}{k}\right)}\left[s \mathcal{L}\{y\}(s)-y\left(0^{+}\right)\right] \\
& \quad \lambda \sum_{i=0}^{\infty} \frac{\beta_{p}(\gamma+i m, d-\gamma)}{\beta(\gamma, d-\gamma)} \frac{(d)_{i m}}{\Gamma(\mu i+v+1)} \frac{w^{i}}{(l)_{i \delta}} \mathcal{L}\left\{x^{\mu i+v}\right\}+\mathcal{L}\{f(x)\} .
\end{aligned}
$$

After simplification we get

$$
\begin{aligned}
y(s)= & \frac{c}{s}+\frac{\lambda k^{1-\alpha} \Gamma_{k}\left(1-\frac{\alpha}{k}\right)}{s^{\mu i+\alpha+v+1} \Gamma_{k}(k-\alpha)} \\
& \times \sum_{i=0}^{\infty} \frac{\beta_{p}(\gamma+i m, d-\gamma)}{\beta(\gamma, d-\gamma)} \frac{(d)_{i m} w^{i}}{(l)_{i \delta}}+\frac{F(s) s^{-\alpha} k^{1-\alpha} \Gamma_{k}\left(1-\frac{\alpha}{k}\right)}{\Gamma_{k}(k-\alpha)} .
\end{aligned}
$$

Now, taking the inverse Laplace transform, we get

$$
\begin{aligned}
y(x)= & c_{1} c+\frac{\lambda k^{1-\alpha} \Gamma_{k}\left(1-\frac{\alpha}{k}\right)}{\Gamma_{k}(k-\alpha)} \mathcal{L}^{-1}\left\{\frac{1}{s^{\mu i+\alpha+\nu+1}}\right\} \\
& \times \sum_{i=0}^{\infty} \frac{\beta_{p}(\gamma+i m, d-\gamma)}{\beta(\gamma, d-\gamma)} \frac{(d)_{i m} w^{i}}{(l)_{i \delta}}+\frac{k^{1-\alpha} \Gamma_{k}\left(1-\frac{\alpha}{k}\right)}{\Gamma_{k}(k-\alpha)} \mathcal{L}^{-1}\left\{F(s) s^{-\alpha}\right\},
\end{aligned}
$$

which gives the required result (3.7). 
Theorem 6 Let $a \in \mathbb{R}^{+}, \mu, v, l, \gamma, d \in \mathbb{C}, \operatorname{Re}(\mu), \operatorname{Re}(v), \operatorname{Re}(l)>0, \operatorname{Re}(d)>\operatorname{Re}(\gamma)>0$ with $p \geq 0, \delta>0$ and $0<m \leq \delta+\operatorname{Re}(\mu)$. The differential equation

$$
\left(C_{k, 0+}^{\alpha} y\right)(x)=\lambda_{1} x^{\nu} E_{\mu, \nu+1, l}^{\gamma, \delta, m, d}\left(w x^{\mu} ; p\right)+\lambda_{2} x^{\nu} E_{\mu, v+1, l}^{\gamma, \delta, m, d}\left(w x^{\mu} ; p\right)
$$

with the initial condition $y\left(0^{+}\right)=c$, where $c$ is an arbitrary constant, has the solution as follows:

$$
\begin{aligned}
y(x)= & c_{1} c+\frac{\left(\lambda_{1}+\lambda_{2}\right) k^{1-\alpha} \Gamma_{k}\left(1-\frac{\alpha}{k}\right)}{\Gamma_{k}(k-\alpha)} \\
& \times \sum_{i=0}^{\infty} \frac{\beta_{p}(\gamma+i m, d-\gamma)}{\beta(\gamma, d-\gamma)} \frac{(d)_{i m}}{\Gamma(\mu i+\alpha+v+1)} \frac{w^{i} x^{\mu i+\alpha+\nu}}{(l)_{i \delta}} .
\end{aligned}
$$

Proof Applying the Laplace transform to equation (3.8), we have

$$
\mathcal{L}\left\{\left(C_{k, 0+}^{\alpha} y\right)(x)\right\}(s)=\left(\lambda_{1}+\lambda_{2}\right) \mathcal{L}\left\{x^{\nu} E_{\mu, v+1, l}^{\gamma, \delta, m, d}\left(w x^{\mu} ; p\right)\right\}(s) .
$$

Using Lemma 2 for $n=1$ and the definition of Mittag-Leffler function, we have

$$
\begin{aligned}
& \frac{\Gamma_{k}(k-\alpha)}{(k s)^{1-\alpha} \Gamma_{k}\left(1-\frac{\alpha}{k}\right)}\left[s \mathcal{L}\{y\}(s)-y\left(0^{+}\right)\right] \\
& \quad=\left(\lambda_{1}+\lambda_{2}\right) \sum_{i=0}^{\infty} \frac{\beta_{p}(\gamma+i m, d-\gamma)}{\beta(\gamma, d-\gamma)} \frac{(d)_{i m}}{\Gamma(\mu i+v+1)} \frac{w^{i}}{(l)_{i \delta}} \mathcal{L}\left\{x^{\mu i+\nu}\right\} .
\end{aligned}
$$

After simplification we get

$$
y(s)=\frac{c}{s}+\frac{\left(\lambda_{1}+\lambda_{2}\right) k^{1-\alpha} \Gamma_{k}\left(1-\frac{\alpha}{k}\right)}{s^{\mu i+\alpha+\nu+1} \Gamma_{k}(k-\alpha)} \sum_{i=0}^{\infty} \frac{\beta_{p}(\gamma+i m, d-\gamma)}{\beta(\gamma, d-\gamma)} \frac{(d)_{i m} w^{i}}{(l)_{i \delta}} .
$$

Now, taking the inverse Laplace transform, we get

$$
\begin{aligned}
y(x)= & c_{1} c+\frac{\left(\lambda_{1}+\lambda_{2}\right) k^{1-\alpha} \Gamma_{k}\left(1-\frac{\alpha}{k}\right)}{\Gamma_{k}(k-\alpha)} \mathcal{L}^{-1}\left\{\frac{1}{s^{\mu i+\alpha+v+1}}\right\} \\
& \times \sum_{i=0}^{\infty} \frac{\beta_{p}(\gamma+i m, d-\gamma)}{\beta(\gamma, d-\gamma)} \frac{(d)_{i m} w^{i}}{(l)_{i \delta}}
\end{aligned}
$$

which gives the required result (3.9).

Theorem 7 Let $a \in \mathbb{R}^{+}, \mu, v, l, \gamma, d \in \mathbb{C}, \operatorname{Re}(\mu), \operatorname{Re}(v), \operatorname{Re}(l)>0, \operatorname{Re}(d)>\operatorname{Re}(\gamma)>0$ with $p \geq 0, \delta>0$ and $0<m \leq \delta+\operatorname{Re}(\mu)$. The differential equation

$$
\left(C_{k, 0+}^{\alpha} y\right)(x)=\lambda x^{\nu} E_{\mu, v+1, l}^{\gamma, \delta, m, d}\left(w x^{\mu} ; p\right)+\sum_{j=1}^{n} \lambda_{j} x^{\nu_{j}} E_{\mu_{j}, \nu_{j}+1, l_{j}}^{\gamma_{j}, \delta_{j}, m_{j}, d_{j}}\left(w_{j}(x)^{\mu_{j}} ; p\right)
$$


with the initial condition $y\left(0^{+}\right)=c$, where $c$ is an arbitrary constant, has the solution as follows:

$$
\begin{aligned}
y(x)= & c_{1} c+\frac{\lambda k^{1-\alpha} \Gamma_{k}\left(1-\frac{\alpha}{k}\right)}{\Gamma_{k}(k-\alpha)} \sum_{i=0}^{\infty}\left[\frac{\beta_{p}(\gamma+i m, d-\gamma)}{\beta(\gamma, d-\gamma)}\right. \\
& \left.\times \frac{(d)_{i m}}{\Gamma(\mu i+\alpha+v+1)} \frac{w^{i} x^{\mu i+\alpha+\nu}}{(l)_{i \delta}}\right]+\sum_{j=1}^{n}\left[\frac{\lambda_{j} k^{1-\alpha} \Gamma_{k}\left(1-\frac{\alpha}{k}\right)}{\Gamma_{k}(k-\alpha)}\right. \\
& \left.\times \sum_{i=0}^{\infty} \frac{\beta_{p}\left(\gamma_{j}+i m_{j}, d_{j}-\gamma_{j}\right)}{\beta\left(\gamma_{j}, d_{j}-\gamma_{j}\right)} \frac{\left(d_{j}\right)_{i m_{j}}}{\Gamma\left(\mu_{j} i+\alpha+v_{j}+1\right)} \frac{w_{j}^{i} x^{\mu j i+\alpha+v_{j}}}{\left(l_{j}\right)_{i \delta_{j}}}\right] .
\end{aligned}
$$

Proof Applying the Laplace transform to the above equation, we have

$$
\begin{aligned}
\mathcal{L}\left\{\left(C_{k, 0+}^{\alpha} y\right)(x)\right\}(s)= & \lambda \mathcal{L}\left\{x^{\nu} E_{\mu, v+1, l}^{\gamma, \delta, m, d}\left(w(x)^{\mu} ; p\right)\right\}(s) \\
& +\mathcal{L}\left\{\sum_{j=1}^{n} \lambda_{j} x^{\nu_{j}} E_{\mu_{j}, \nu_{j}+1, l_{j}}^{\gamma_{j}, \delta_{j}, m_{j}, d_{j}}\left(w_{j}(x)^{\mu_{j}} ; p\right)\right\}(s) .
\end{aligned}
$$

Using Lemma 2 for $n=1$ and the definition of Mittag-Leffler function, we have

$$
\begin{aligned}
& \frac{\Gamma_{k}(k-\alpha)}{(k s)^{1-\alpha} \Gamma_{k}\left(1-\frac{\alpha}{k}\right)}\left[s \mathcal{L}\{y\}(s)-y\left(0^{+}\right)\right] \\
& =\lambda \sum_{i=0}^{\infty} \frac{\beta_{p}(\gamma+i m, d-\gamma)}{\beta(\gamma, d-\gamma)} \frac{(d)_{i m}}{\Gamma(\mu i+v+1)} \frac{w^{i}}{(l)_{i \delta}} \mathcal{L}\left\{x^{\mu i+\nu}\right\} \\
& \quad+\sum_{j=1}^{n} \lambda_{j}\left[\sum_{i=0}^{\infty} \frac{\beta_{p}\left(\gamma_{j}+i m_{j}, d_{j}-\gamma_{j}\right)}{\beta\left(\gamma_{j}, d_{j}-\gamma_{j}\right)} \frac{\left(d_{j}\right)_{i m_{j}}}{\Gamma\left(\mu_{j} i+v_{j}+1\right)} \frac{w_{j}^{i}}{\left(l_{j}\right)_{i \delta_{j}}} \mathcal{L}\left\{x^{\mu_{j} i+v_{j}}\right\}\right] .
\end{aligned}
$$

After simplification we get

$$
\begin{aligned}
y(s)= & \frac{c}{s}+\frac{\lambda k^{1-\alpha} \Gamma_{k}\left(1-\frac{\alpha}{k}\right)}{s^{\mu i+\alpha+\nu+1} \Gamma_{k}(k-\alpha)} \sum_{i=0}^{\infty} \frac{\beta_{p}(\gamma+i m, d-\gamma)}{\beta(\gamma, d-\gamma)} \frac{(d)_{i m} w^{i}}{(l)_{i \delta}} \\
& +\sum_{j=1}^{n}\left[\frac{\lambda_{j} k^{1-\alpha} \Gamma_{k}\left(1-\frac{\alpha}{k}\right)}{s^{\mu_{j} i+\alpha+v_{j}+1} \Gamma_{k}(k-\alpha)} \sum_{i=0}^{\infty} \frac{\beta_{p}\left(\gamma_{j}+i m_{j}, d_{j}-\gamma_{j}\right)}{\beta\left(\gamma_{j}, d_{j}-\gamma_{j}\right)} \frac{\left(d_{j}\right)_{i m_{j}} w_{j}^{i}}{\left(l_{j}\right)_{i \delta_{j}}}\right] .
\end{aligned}
$$

Now, taking the inverse Laplace transform, we get

$$
\begin{aligned}
y(x)= & c_{1} c+\frac{\lambda k^{1-\alpha} \Gamma_{k}\left(1-\frac{\alpha}{k}\right)}{\Gamma_{k}(k-\alpha)} \mathcal{L}^{-1}\left\{\frac{1}{s^{\mu i+\alpha+\nu+1}}\right\} \\
& \times \sum_{i=0}^{\infty} \frac{\beta_{p}(\gamma+i m, d-\gamma)}{\beta(\gamma, d-\gamma)} \frac{(d)_{i m} w^{i}}{(l)_{i \delta}} \\
& +\sum_{j=1}^{n}\left[\frac{\lambda_{j} k^{1-\alpha} \Gamma_{k}\left(1-\frac{\alpha}{k}\right)}{\Gamma_{k}(k-\alpha)} \mathcal{L}^{-1}\left\{\frac{1}{s^{\mu_{j} i+\alpha+\nu_{j}+1}}\right\} \sum_{i=0}^{\infty} \frac{\beta_{p}\left(\gamma_{j}+i m_{j}, d_{j}-\gamma_{j}\right)}{\beta\left(\gamma_{j}, d_{j}-\gamma_{j}\right)} \frac{\left(d_{j}\right)_{i m_{j}} w_{j}^{i}}{\left(l_{j}\right)_{i \delta_{j}}}\right],
\end{aligned}
$$

which gives the required result (3.11). 


\subsection{Concluding remarks}

In this paper, by using Grüss type variable bounds of the functions having $n$th derivatives absolutely continuous, Caputo $k$-fractional integral inequalities have been established. Integral inequalities have been derived deploying Chebyshev inequality [12] for Caputo $k$-fractional derivatives. Laplace transform of Caputo $k$-fractional derivative is derived and further applied to solve fractional differential equations involving an extended generalized Mittag-Leffler function. Caputo $k$-fractional derivative and Riemann-Liouville $k$-fractional integral of the extended generalized Mittag-Leffler function are calculated.

\section{Acknowledgements}

We thank to the editor and referees for their careful reading and valuable suggestions to make the article friendly readable. The research work of Ghulam Farid is supported by the Higher Education Commission of Pakistan under NRPU 2016, Project No. 5421

\section{Funding}

Not applicable.

\section{Competing interests}

The authors declare that they have no competing interests.

\section{Authors' contributions}

All authors have equal contribution in this article. All authors read and approved the final manuscript.

\section{Author details}

'Department of Mathematics, COMSATS University Islamabad, Attock, Pakistan. ${ }^{2}$ General Studies Department, Jubail Industrial College, Jubail Industrial City, Jubail, Kingdom of Saudi Arabia. ${ }^{3}$ School of Natural Sciences, National University of Sciences and Technology, Islamabad, Pakistan.

\section{Publisher's Note}

Springer Nature remains neutral with regard to jurisdictional claims in published maps and institutional affiliations.

Received: 15 July 2019 Accepted: 4 October 2019 Published online: 16 October 2019

\section{References}

1. Andrić, M., Farid, G., Pečarić, J.: A further extension of Mittag-Leffler function. Fract. Calc. Appl. Anal. 21(5), 1377-1395 (2018)

2. Caputo, M.: Linear models of dissipation whose $\mathrm{Q}$ is almost frequency independent II. Geophys. J. R. Astron. Soc. 13, 529-539 (1967)

3. Diaza, R., Pariglan, E.: On hypergeometric function and k-pochemer. Divulg. Mat. 15(2), 179-192 (2007)

4. Farid, G., Javed, A.: On Hadamard and Fejér-Hadamard inequalities for Caputo $k$-fractional derivatives. Int. J. Nonlinear Anal. Appl. 9(1), 69-81 (2018)

5. Farid, G., Javed, A., Rehman, A.U.: On Hadamard inequalities for $n$-times differentiable functions which are relative convex via Caputo $k$-fractional derivatives. Nonlinear Anal. Forum 22(2), 17-28 (2017)

6. Farid, G., Javed, A., Rehman, A.U.: Fractional integral inequalities of Hadamard-type for $m$-convex functions via Caputo $k$-fractional derivatives. J. Fract. Calc. Appl. 10(1), 120-134 (2019)

7. Kilbas, A.A., Srivastava, H.M., Trujillo, J..: Theory and Applications of Fractional Differential Equations. North-Holland Mathematics Studies, vol. 204. Elsevier, Amsterdam (2006)

8. Mishra, L.N., Ain, Q.U., Farid, G., Rehman, A.U.: $k$-Fractional integral inequalities for $(h-m)$-convex functions via Caputo $k$-fractional derivatives. Korean J. Math. 27(2), 355-372 (2019)

9. Mittag-Leffler, G.: Sur la nouvelle fonction $E_{\alpha}(x)$. C. R. Acad. Sci., Paris 137, 554-558 (1903)

10. Mubeen, S., Habibullah, G.M.: k-Fractional integrals and application. Int. J. Contemp. Math. Sci. 7(2), 89-94 (2012)

11. Waheed, A., Farid, G., Rehman, A.U., Ayub, W.: k-Fractional integral inequalities for harmonically convex functions via Caputo $k$-fractional derivatives. Bull. Math. Anal. Appl. 10(1), 55-67 (2018)

12. Waheed, A., Rehman, A.U., Qureshi, M.I., Shah, F.A., Khan, K.A., Farid, G.: On Caputo k-fractional derivatives and associated inequalities. IEEE Access 7, 32137-32145 (2019) 